\title{
BMJ Open Effectiveness of non-pharmacological strategies in the management of type 2 diabetes in primary care: a protocol for a systematic review and network meta- analysis
}

\author{
Renata Giacomini Oliveira Ferreira Leite, ${ }^{1}$ Luísa Rocco Banzato, ${ }^{1}$ \\ Julia Simões Corrêa Galendi, ${ }^{1}$ Adriana Lucia Mendes, ${ }^{1}$ Fernanda Bolfi, ${ }^{1}$ \\ Areti Angeliki Veroniki (1) ,2,3,4 Lehana Thabane, ${ }^{5,6}$ \\ Vania dos Santos Nunes-Nogueira (i) ${ }^{1}$
}

To cite: Leite RGOF, Banzato LR, Galendi JSC, et al. Effectiveness of non-pharmacological strategies in the management of type 2 diabetes in primary care: a protocol for a systematic review and network meta-analysis. BMJ Open 2020;10:e34481. doi:10.1136/ bmjopen-2019-034481

- Prepublication history and additional material for this paper are available online. To view these files, please visit the journal online (http://dx.doi org/10.1136/bmjopen-2019034481).

Received 21 September 2019 Revised 26 November 2019 Accepted 10 December 2019

A Check for updates

(c) Author(s) (or their employer(s)) 2020. Re-use permitted under CC BY-NC. No commercial re-use. See rights and permissions. Published by BMJ.

For numbered affiliations see end of article.

Correspondence to Professor Vania dos Santos Nunes-Nogueira;

vania.nunes-nogueira@unesp.br

\section{ABSTRACT}

Introduction Despite the increasing number of drugs and various guidelines on the management of type 2 diabetes mellitus (T2DM), several patients continue with the disease uncontrolled. There are several non-pharmacological treatments available for managing T2DM, but various of them have never been compared directly to determine the best strategies.

Objective This study will evaluate the comparative effects of non-pharmacological strategies in the management of T2DM in primary care or community settings.

Methods and analysis We will perform a systematic review and network meta-analysis (NMA), and will include randomised controlled trials if one of the following interventions were applied in adult patients with T2DM: nutritional therapy, physical activity, psychological interventions, social interventions, multidisciplinary lifestyle interventions, diabetes self-management education and support (DSMES), technology-enabled DSMES, interventions delivered only either by pharmacists or by nurses, self-blood glucose monitoring in non-insulin-treated T2DM, health coaching, benchmarking and usual care. The primary outcome will be glycaemic control (glycated haemoglobin ( $\mathrm{HbA1c})(\%)$ ), and the secondary outcomes will be weight loss, quality of life, patient satisfaction, frequency of cardiovascular events and deaths, number of patients in each group with $\mathrm{HbA} 1 \mathrm{C}<7$, adverse events and medication adherence. We have developed search strategies for Embase, Medline, Latin American and Caribbean Health Sciences Literature, Cochrane Central Register of Controlled Trials, Trip database, Scopus, Web of Science, Cumulative Index to Nursing and Allied Health Literature Australasian Medical Index and Chinese Biomedical Literature Database. Four reviewers will assess the studies for their eligibility and their risk of bias in pairs and independently. An NMA will be performed using a Bayesian hierarchical model, and the treatment hierarchy will be obtained using the surface under the cumulative ranking curve. To determine our confidence in an overall treatment ranking from the NMA, we will follow the
Strengths and limitations of this study

Network meta-analysis (NMA) allows the simultaneous comparison of multiple treatment alternatives in a single model.

- NMA improves precision of treatment effect estimates, ranks treatments according to their effectiveness and can assess the impact of observed treatment effects in the evidence network.

- A potential limitation of this study can be missing outcome data, which may bias our findings. In such a case, valid imputation methods will be applied and robustness of results will be explored.

- Intransitivity in indirect comparisons may be another potential limitation, which can impact the validity of our NMA results. In case of intransitivity, reasons for this will be explored.

Grading of Recommendations Assessment, Development and Evaluation approach.

Ethics and dissemination As no primary data collection will be undertaken, no formal ethical assessment is required. We plan to present the results of this systematic review in a peer-reviewed scientific journal, conferences and the popular press.

PROSPERO registration number CRD42019127856.

\section{INTRODUCTION}

Despite the increasing number of drugs available and various guidelines on the management of type 2 diabetes mellitus (T2DM), an expressive number of patients continue with the disease uncontrolled. In a multicentre, cross-sectional, epidemiological, questionnaire-based study conducted in nine Latin American countries, 56.8\% of patients with T2DM had poor glycaemic control (haemoglobin A1c (HbAlc) $\geq 7 \%)$. 
In the USA, according to a survey performed between 1998 and 2002, only $42.3 \%$ of adults had HbA1c levels less than $7 \%$, and $14 \%$ had HbA1c levels greater than $10 \% .^{2}$

Therefore, to increase the percentage of diabetic patients with the disease controlled and thereby reduce the number of deaths and morbidities related to this disease, non-pharmacological strategies that are complementary to the drug treatment have been studied in the management of T2DM.

Randomised clinical trials (RCTs) have shown that medical nutritional therapy and physical activity, considered as non-pharmacological treatments of T2DM, effectively improve glycaemic control and other metabolic outcomes in patients with T2DM. ${ }^{34}$ Additionally, a systematic review of lifestyle weight loss interventions in overweight and obese adults with T2DM showed that a weight loss of $>5 \%$ is considered necessary for its beneficial effects on HbAlc, lipids and blood pressure, and to achieve this level of weight loss, intense interventions, including energy restriction, regular physical activity and frequent contact with healthcare professionals, are required. ${ }^{5}$

Meanwhile, other non-pharmacological strategies have been introduced in diabetes treatment. Some studies in T2DM have shown that programmes focused on counselling, therapy compliance, explanation of possible adverse events and patient empowerment are associated with better glycaemic and quality-of-life controls and, consequently, lower follow-up costs. ${ }^{6-9}$ A systematic review of the effects of group-based, patient-centred training on clinical, lifestyle and psychosocial outcomes in patients with T2DM showed significant reductions in HbA1c in favour of group-based interventions. ${ }^{10}$ Similarly, other strategies with similar objectives have also been reported in the management of T2DM, such as psychological ${ }^{11}$ and social interventions. ${ }^{12}$

However, despite the evidence of effectiveness of these non-pharmacological strategies in T2DM metabolic control, in primary healthcare settings, some RCTs have not achieved similar results. ${ }^{13-15}$ In a pragmatic clustered randomised controlled trial conducted in public community health centres in Cape Town involving 1570 adults with T2DM, a group diabetes education programme did not show greater improvement in glycaemia control compared with usual care. ${ }^{16}$

Since there are several different non-pharmacological strategies for the management of T2DM and with contradictory results in some healthcare settings, we aim to answer the following questions: in primary care, are the non-pharmacological strategies effective in the glycaemic control of adults with T2DM? Which of these strategies have the best glycaemic control?

Hence, the objective of this study is to evaluate the comparative effects of non-pharmacological strategies in the management of T2DM in primary care or community settings.

\section{METHODS AND DESIGN}

A systematic review and network meta-analysis (NMA) for the assessment of the effectiveness of all nonpharmacological strategies available for T2DM in diabetes control will be performed.

NMA combines direct and indirect evidence; therefore, the relative effectiveness of two non-pharmacological strategies can be estimated even if studies that directly compared them did not exist.

Denoting nutritional therapy, social support and usual care as non-pharmacological strategies A, B and C, respectively, an indirect comparison $(\mathrm{AB})$ can be obtained by subtracting the meta-analytic estimates of all studies of nutritional therapy versus usual care (AC) from the estimate of all studies of social support versus usual care (BC). ${ }^{17}$

Traditional meta-analyses are limited to the comparisons of two groups, failing to generate a complete picture of the effectiveness of non-pharmacological treatments for T2DM. In the current review, since there are more than 10 strategies of interest and for most there are no trials involving a direct comparison, the NMA was selected a substitute of the traditional meta-analysis.

The protocol of this review has been registered with the International Prospective Register of Systematic Reviews database, and it was developed following the Preferred Reporting Items for Systematic Review and Meta-Analysis Protocols. ${ }^{18}$

\section{Patient and public involvement}

We will not directly include patient-level data in this study, but the protocol development, priority of the research question, choice of outcome measures and type of intervention have been informed through discussions with the members of the Brazilian Health Ministry and a group of patients with T2DM during follow-up in a tertiary Brazilian healthcare; both identified this study as a priority area for managing patients with T2DM in primary healthcare.

\section{Eligibility criteria}

RCTs meeting the 'PICOT' structure described below will be included in this study.

\section{Participants (P)}

Adult patients, over 18 years old, diagnosed with T2DM according to the American Diabetes Association (fasting glycaemia greater than or equal to $126 \mathrm{mg} / \mathrm{dL}$, glycaemia greater than $200 \mathrm{mg} / \mathrm{dL}$ associated with classic DM symptoms, glycaemia 2 hours after overload with $75 \mathrm{~g}$ of glucose greater than or equal to $200 \mathrm{mg} / \mathrm{dL}$, HbAlc greater than or equal to $6.5 \%$ ) will be included in the study. ${ }^{6}$

\section{Definitions of interventions (l)}

All non-pharmacological and patient-mediated strategies ${ }^{19}$ aimed at promoting better control of the disease for diabetic patients will be considered as interventions. The strategies can be implemented as either standalone or adjunct to the pharmacotherapy of T2DM. Regarding 
adjunct treatment, both groups must have received similar drug treatment.

Based on our previous search in the literature, the interventions may be (1) nutritional therapy (dietary quality or energy restriction), ${ }^{20}$ (2) physical activity programme (running, walking, bicycling, swimming, resistance training, yoga, Tai chi), ${ }^{20}$ (3) psychological interventions (emotion-focused or cognition-focused), ${ }^{11}$ (4) social network interventions (friends, families and peers), ${ }^{12}$ (5) multidisciplinary lifestyle interventions (an intervention that combines at least two of the following modalities: physical activity, nutritional therapy, social or psychological support), ${ }^{21}$ (6) diabetes self-management education and support (DSMES), ${ }^{22}$ (7) technology-enabled DSMES (mobile phones, secure messaging, web-based information) ${ }^{23}(8)$ interventions delivered only or mainly by pharmacists (DSMES and/or pharmacy management), ${ }^{24}$ (9) interventions delivered only or mainly by nurses (DSMES and/or pharmacy management), ${ }^{25}$ (10) selfblood glucose monitoring in non-insulin-treated T2DM, ${ }^{26}$ (11) health coaching ${ }^{27}$ and (12) benchmarking. ${ }^{28}$

The intervention must have been performed at the primary care (or in community settings), with a minimum follow-up period of 6 months.

\section{Comparison (C)}

Comparator will be considered a usual care of T2DM (drug treatment associated with a general orientation regarding lifestyle changes provided by a general practitioner) or another intervention described above. An episodic evaluation with a nutritionist, nurse, physical trainer or educator in diabetes, which provides a general orientation regarding changes in lifestyle, will be considered usual care if the patients are not provided with subsequent follow-up.

This protocol differs from our previous published protocol ${ }^{29}$ because in the current systematic review, we will consider all non-pharmacological strategies for T2DM in primary care. Additionally, here, we will perform direct and indirect comparisons of all strategies. In the previous protocol, only nutritional therapy has been evaluated in direct comparisons (only nutritional therapy vs usual care).

\section{Type of outcomes (0)}

The primary outcome will be glycaemic control (HbAlc $(\%)$ ). The secondary outcomes will be anthropometric measurements (measured by weight or waist circumference (WC), or body mass index (BMI)), quality of life, patient satisfaction, frequency of cardiovascular events and deaths, number of patients in each group with HbA1c $<7$, adverse events related to non-pharmacological strategies and medication adherence.

\section{Time-frame of outcome evaluation (T)}

We will include only studies with follow-up greater than 6 months. The outcomes will be evaluated at 6-12 months and greater than 12 months. For trials that had more than one time of outcome evaluation, we will consider the longest time point.

\section{Exclusion criteria}

We will exclude trials that were conducted in settings other than the primary care or community settings, trials whose aim was to compare the effectiveness of pharmacological treatments, trials in which the intervention was any type of surgery to lose weight, trials with follow-up period less than 6 months and trials that included predominantly participants with type $1 \mathrm{DM}$, gestational diabetes, or diabetes secondary to medication or a chronic disease.

\section{Data sources and search strategy}

Search strategies have been created and adapted to the following electronic health databases: Embase (by Elsevier, 1980-2019), Medline (by PubMed, 1966-2019), Latin American and Caribbean Health Sciences Literature (by Virtual Health Library, 1982-2019) and Controlled Clinical Trials of the Cochrane Collaboration (Cochrane Central Register of Controlled Trials). We have used the following index terms and their synonyms: Diabetes Mellitus, Type 2; Primary Health Care; Community Health Planning. Language or year restrictions will not be considered in this study. We have used the validated RCT filters created by the Cochrane Collaboration for Medline and Embase. A draft Medline search strategy is included in online supplementary appendix 1 .

The following databases will also be searched for eligible studies: Trip database, Scopus, Web of Science, Cumulative Index to Nursing and Allied Health Literature, Australasian Medical Index and Chinese Biomedical Literature Database. We will also search for studies on ClinicalTrials.gov and the gray literature through conferences, published abstracts and dissertations.

References of relevant primary or secondary studies will be searched to identify additional eligible studies. We will use the Endnote software to download all references and remove duplicates. The initial screening of abstracts and titles will be performed using the free web application Rayyan QCRI. ${ }^{30}$

\section{Study selection}

Four reviewers independently will perform in pairs the assessment of titles and abstracts (RGOFL, LRB, JSCG, VdSN-N), and the studies potentially eligible for inclusion in the review will be selected for full reading and subsequently assessed for adequacy to the proposed PICOT. In case of disagreement, a consensus meeting before the final decision will be held.

\section{Data extraction}

For each selected trial, the same four reviewers will use in pairs and independently an extraction form to record the year of publication, number of patients included, duration of follow-up, information regarding the inclusion and exclusion criteria, type of intervention (frequency, descriptions, durations), baseline data (average age, gender, weight, BMI and WC, glycaemic control prior to the study, 
duration of T2DM, medications in use) and all reported outcome measures (in all time points). To ensure consistency between the reviewers, we will perform a calibration exercise before beginning the review. In the case of duplicate publications or more reports from the primary trial, data extraction will be optimised using the best information available for all the items in the same trial.

\section{Assessment of bias risk in the included studies}

For each selected trial, the risk of bias will be assessed according to the criteria described in the revised Cochrane risk-of-bias tool for randomised trials (RoB 2 tool), ${ }^{31}$ which considers the following five domains for each outcome evaluated: (1) bias arising from the randomisation process, (2) bias due to deviations from intended interventions, (3) bias due to missing outcome data, (4) bias in the measurement of the outcome and (5) bias in the selection of the reported result. Each of the items will be evaluated by two reviewers as having low risk of bias, some concerns and high risk of bias. In case of disagreement, a discussion between the reviewers before the final classification will be held.

\section{Data synthesis}

Dealing with missing data

The authors of the original studies will be contacted, if necessary, to provide missing information for each study included. We will use the data available in published articles provided by their authors or registration platforms. If available, we will preferentially use the data from intentionto-treat analysis. If numerical outcome data are missing and they cannot be obtained from the authors, we will calculate them, when possible, from other available statistics, such as $\mathrm{p}$ values. ${ }^{32}$ If an outcome value is reported without a measure of variance, SDs will be imputed according to the method suggested by Furukawa $e t$ al. ${ }^{33}$

\section{Assessment of transitivity across treatment comparisons}

The transitivity across treatment comparisons will be assessed using boxplots, and we are proposing the following seven a priori hypotheses to explain the variability between studies as possible effect modifiers: (1) patient characteristics (average patient age, gender distribution, disease severity, time of diabetes diagnosis, presence of diabetes chronic complications), (2) type of pharmacological treatment of T2DM, (3) study methodology quality (low risk of bias compared with high risk of bias), sample size (large vs small studies), (4) duration of follow-up (6-12 months, greater than 12 months), (5) frequency of sessions/visits with participants and (6) adherence to a healthier lifestyle. Usual care of T2DM will be assessed for their similarity across treatment comparisons. ${ }^{34}$

\section{Network meta-analysis}

We will perform an NMA for each outcome to simultaneously compare multiple interventions in a single model using the Stata Statistical Software V.16 (StataCorp LLC).
We will preferentially pool the direct evidence; however, in the absence of direct comparisons, the effect estimate will be provided by indirect comparisons.

Considering the expected between-study heterogeneity, we will use a random effects (RE) model for each intervention comparison.

We will pool the data of each outcome using a Bayesian RE model separately. For dichotomous data, effect estimates will be calculated using OR with a $95 \%$ credible interval (CrI). The continuous data will be expressed as means and SDs for each study, and the mean difference or standardised mean difference (if different metrics are used across studies) will be calculated with their respective $95 \%$ CrIs. For count outcomes, we will calculate the rate ratio with a $95 \%$ CrI. For multiarm studies, we plan to use data from all reported comparisons using the approach suggested by Rücker et al by reducing the relevant weighting scheme. ${ }^{35}$

The intervention effect estimates will be presented along with their corresponding 95\% CrIs, and we will obtain the treatment hierarchy using the surface under the cumulative ranking (SUCRA) curve, with its $95 \% \mathrm{CrI}$, and the rank-heat plot. ${ }^{36}{ }^{37}$ It is expected that the best treatment will have high SUCRA values while the worst will have low values. For each comparison, we will present the direct, indirect and network estimates.

\section{Assessment of statistical heterogeneity}

For direct evidence, we will assess heterogeneity by estimating the magnitude of the between-study variance using the empirical distribution as estimated by Turner et $a l^{88}$ and Rhodes $e t a l^{39}$ and by using the $\mathrm{I}^{2}$ statistic to quantify the percentage of variability due to true differences between studies rather than sampling error. ${ }^{40}{ }^{41}$ We will interpret the $\mathrm{I}^{2}$ according to thresholds set forth by the Cochrane Collaboration, ${ }^{32}$ and it will be used as a criterion for pooling or not the results and for performing additional subgroup analyses. For count outcomes, we will use a minimally informative prior distribution $(\sim$ Uniform $[0,2]){ }^{42}$

If enough studies are available, we will perform subgroup analysis using the same potential treatment effect modifiers described above. Our a priori hypothesis is as follows: individuals with greater than 10 years of T2DM, taking insulin, with a poorly controlled diabetes at baseline (an uninterrupted HbA1c $>8.0 \%$ for $\geq 1$ year despite standard care) and with more than one of the macro or micro chronic diabetes complications, the subgroups analysis may show less improvement in the primary and secondary outcomes. We will also perform a network meta-regression whenever possible (ie, when at least 10 studies are available) using the RE model to evaluate the impact of these potential effect modifiers (patient characteristic, study quality, intervention type, follow-up time, adherence).

With the combination of direct and indirect estimates, violation of the transitivity assumption (described above) will also lead to inconsistency. We will assess loop 
inconsistency (disagreement between direct and indirect estimates) using the loop-specific method and design inconsistency (disagreement between studies that inform the same treatment comparison but include a different number of treatment arms) using the design-by-treatment model based on a $\chi^{2}$ test. $^{43-46}$

\section{Sensitivity analysis}

If sufficient studies are available, we will conduct a sensitivity analysis to assess the robustness of results. ${ }^{38} 39$ This analysis will be performed by comparison of studies with high risk of selection and attrition bias versus studies with low risk of bias in these domains and studies with data published versus studies with imputed data.

\section{Assessment of publication biases}

For each treatment comparison, if more than 10 studies are included in the meta-analysis, we will use the funnel plot to investigate the presence of publication bias. ${ }^{32}$ In such cases, we will also perform the Begg's rank correlation $^{47}$ and Egger's regression tests. ${ }^{48}$

\section{Quality of evidence}

To determine our confidence in an overall treatment ranking from the NMA, we will follow the Grading of Recommendations Assessment, Development and Evaluation (GRADE) approach, with some modifications as described below to reflect specific issues from NMA. ${ }^{49}$ This process will be performed in pairs and independently (RGOFL, LRB, JSCG, VdSN-N).

Based on the five categories (risk of bias, imprecision, inconsistency and publication bias) the certainty of evidence of effect estimates obtained by direct comparisons will be rated as high, moderate, low or very low.

For indirect comparisons, the quality of evidence in estimates will be rated following the GRADE categories used for assessing the direct comparisons in addition to the transitivity assessment. We will focus our assessments on the quality of indirect evidence on the dominant first-order loop (loops with a single common comparator connecting the two interventions of the comparison of interest). The quality of evidence rating for indirect comparisons will be the lower ratings of quality for the two direct estimates that contribute to the first-order loop of the indirect comparison. For instance, if one of the direct comparisons is rated as low and the other is rated as moderate evidence, we will rate the quality of indirect evidence as low. ${ }^{45}$ We will rate down the quality of the indirect comparison one further level for violation of the transitivity assumption (similarity of trials in terms of population, intervention (type and dosing frequency), settings and trial methodology). ${ }^{45}$

We will rate the confidence in each NMA effect estimate using the higher rating when both direct and indirect evidences are present. However, we may rate down confidence in the network estimate if we find that the direct and indirect estimates have inconsistency (measured by the difference of point estimates and the extent of overlap of CrIs and of direct and indirect effect estimates).

\section{DISCUSSION}

With the consistent increase in the prevalence of T2DM together with the unsatisfactory glycaemic control by some individuals, the search for new and effective strategies for the prevention and control of this metabolic disease is underway.

Since inadequate glycaemic control in diabetes is most often related to poor adherence to lifestyle changes and to the proposed treatment, initiatives have emerged to promote a better acceptance/understanding of the disease and its treatment by the patients. With this, it is expected that individuals have a more active participation in the control of his, disease, thus achieving higher rates of glycaemic control and fewer complications associated with this dysglycaemia.

Although several systematic reviews have evaluated the effectiveness of these strategies in the management of $\mathrm{T} 2 \mathrm{DM},{ }^{850}$ to the best of our knowledge to date, there are no systematic reviews and NMA considering the direct and indirect effects of non-pharmacological interventions targeting a greater control of T2DM.

\section{ETHICS AND DISSEMINATION}

Since primary data collection will be undertaken, no formal ethical assessment is required by our institution. We plan to present the findings of this systematic review in a peer-reviewed scientific journal. We also intend to present it, including preliminary findings, at the appropriate conferences.

\section{Author affiliations}

${ }^{1}$ Department of Internal Medicine, São Paulo State University/UNESP, Medical School, Botucatu, São Paulo, Brazil

${ }^{2}$ Department of Primary Education, School of Education, University of loannina, Ioannina, Greece

${ }^{3}$ Knowledge Translation Program, Li Ka Shing Knowledge Institute, St. Michael's Hospital, Unity Health Toronto, Toronto, ON, Canada

${ }^{4}$ Institute of Reproductive and Developmental Biology, Department of Surgery \& Cancer, Faculty of Medicine, Imperial College, London, United Kingdom

${ }^{5}$ Department of Health Research Methods, Evidence, and Impact, McMaster University, Hamilton, ON, Canada

${ }^{6}$ Departments of Pediatrics and Anesthesia, McMaster University, Hamilton, ON, Canada

Twitter Vania dos Santos Nunes-Nogueira @vsnunes

Contributors VdSN-N, LT and A-AV conceptualised and design the study. LT, RGOFL, A-AV and VdSN-N drafted the manuscript protocol. VdSN-N, RGOFL, LRB, ALM, JSCG, FB, A-AV and LT critically revised the protocol and manuscript submitted. All authors read and approved the final manuscript.

Funding This research has been partially supported by the São Paulo Research Foundation (Grant number: 2018/25035-5), and A-AV is funded by the European Union's Horizon 2020 (No. 754936).

Competing interests None declared.

Patient consent for publication Not required.

Provenance and peer review Not commissioned; externally peer reviewed. 
Open access This is an open access article distributed in accordance with the Creative Commons Attribution Non Commercial (CC BY-NC 4.0) license, which permits others to distribute, remix, adapt, build upon this work non-commercially, and license their derivative works on different terms, provided the original work is properly cited, appropriate credit is given, any changes made indicated, and the use is non-commercial. See: http://creativecommons.org/licenses/by-nc/4.0/.

\section{ORCID iDs}

Areti Angeliki Veroniki http://orcid.org/0000-0001-6388-4825

Vania dos Santos Nunes-Nogueira http://orcid.org/0000-0001-9316-4167

\section{REFERENCES}

1 Lopez Stewart G, Tambascia M, Rosas Guzmán J, et al. Control of type 2 diabetes mellitus among general practitioners in private practice in nine countries of Latin America. Rev Panam Salud Publica 2007;22:12-20.

2 Saaddine JB, Cadwell B, Gregg EW, et al. Improvements in diabetes processes of care and intermediate outcomes: United States, 1988-2002. Ann Intern Med 2006;144:465-74.

3 Franz MJ, MacLeod J. Success of nutrition-therapy interventions in persons with type 2 diabetes: challenges and future directions. Diabetes Metab Syndr Obes 2018;11:265-70.

4 Gregg EW, Chen H, Wagenknecht LE, et al. Association of an intensive lifestyle intervention with remission of type 2 diabetes. JAMA 2012;308:2489-96.

5 Franz MJ, Boucher JL, Rutten-Ramos S, et al. Lifestyle WeightLoss Intervention Outcomes in Overweight and Obese Adults with Type 2 Diabetes: A Systematic Review and Meta-Analysis of Randomized Clinical Trials. J Acad Nutr Diet 2015;115:1447-63.

6 American Diabetes A. Standards of medical care in Diabetes-2017 abridged for primary care providers. Clin Diabetes 2017;35:5-26.

7 Jacob S, Serrano-Gil M. Engaging and empowering patients to manage their type 2 diabetes, part II: initiatives for success. Adv Ther 2010;27:665-80.

8 Serrano-Gil M, Jacob S. Engaging and empowering patients to manage their type 2 diabetes, part I: a knowledge, attitude, and practice gap? Adv Ther 2010;27:321-33.

9 Chapman A, Liu S, Merkouris S, et al. Psychological interventions for the management of glycemic and psychological outcomes of type 2 diabetes mellitus in China: a systematic review and meta-analyses of randomized controlled trials. Front Public Health 2015;3:252.

10 Odgers-Jewell K, Ball LE, Kelly JT, et al. Effectiveness of groupbased self-management education for individuals with type 2 diabetes: a systematic review with meta-analyses and metaregression. Diabet Med 2017;34:1027-39.

11 Chew BH, Vos RC, Metzendorf M-I, et al. Psychological interventions for diabetes-related distress in adults with type 2 diabetes mellitus. Cochrane Database Syst Rev 2017;39:CD011469.

12 Spencer-Bonilla G, Ponce OJ, Rodriguez-Gutierrez R, et al. A systematic review and meta-analysis of trials of social network interventions in type 2 diabetes. BMJ Open 2017;7:e016506.

13 Browning C, Chapman A, Yang H, et al. Management of type 2 diabetes in China: the happy life Club, a pragmatic cluster randomised controlled trial using health coaches. BMJ Open 2016;6:e009319.

14 Liss DT, Finch EA, Cooper A, et al. One-year effects of a group-based lifestyle intervention in adults with type 2 diabetes: a randomized encouragement trial. Diabetes Res Clin Pract 2018:140:36-44.

15 Edelman D, Dolor RJ, Coffman CJ, et al. Nurse-Led behavioral management of diabetes and hypertension in community practices: a randomized trial. J Gen Intern Med 2015;30:626-33.

16 Mash RJ, Rhode $\mathrm{H}$, Zwarenstein M, et al. Effectiveness of a group diabetes education programme in under-served communities in South Africa: a pragmatic cluster randomized controlled trial. Diabet Med 2014;31:987-93.

17 Cipriani A, Higgins JPT, Geddes JR, et al. Conceptual and technical challenges in network meta-analysis. Ann Intern Med 2013;159:130-7.

18 Shamseer L, Moher D, Clarke M, et al. Preferred reporting items for systematic review and meta-analysis protocols (PRISMA-P) 2015: elaboration and explanation. BMJ 2015;349:97647.

19 Effective Practice and Organisation of Care (EPOC). Epoc taxonomy, 2015. Available: https://epoc.cochrane.org/epoc-taxonomy [Accessed 4 Jul 2019].

20 Davies MJ, D'Alessio DA, Fradkin J, et al. Management of hyperglycemia in type 2 diabetes, 2018. A consensus report by the American diabetes association (ADA) and the European association for the study of diabetes (EASD). Diabetes Care 2018;41:2669-701.
21 Johansen MY, MacDonald CS, Hansen KB, et al. Effect of an intensive lifestyle intervention on glycemic control in patients with type 2 diabetes. JAMA 2017;318:637-46.

22 Chatterjee S, Davies MJ, Heller S, et al. Diabetes structured selfmanagement education programmes: a narrative review and current innovations. Lancet Diabetes Endocrinol 2018;6:130-42.

23 Greenwood DA, Gee PM, Fatkin KJ, et al. A systematic review of reviews evaluating Technology-Enabled diabetes self-management education and support. J Diabetes Sci Technol 2017;11:1015-27.

24 Aubert REet al. Nurse case management to improve glycemic contro in diabetic patients in a health maintenance organization. Ann Intern Med 1998;129:605-12

25 Azami G, Soh KL, Sazlina SG, et al. Effect of a nurse-led diabetes self-management education program on glycosylated hemoglobin among adults with type 2 diabetes. J Diabetes Res 2018;2018:1-12.

26 Zhu H, Zhu Y, Leung S-wai. Is self-monitoring of blood glucose effective in improving glycaemic control in type 2 diabetes without insulin treatment: a meta-analysis of randomised controlled trials. BMJ Open 2016;6:e010524.

27 Willard-Grace R, Chen EH, Hessler D, et al. Health coaching by medical assistants to improve control of diabetes, hypertension, and hyperlipidemia in low-income patients: a randomized controlled trial. Ann Fam Med 2015;13:130-8.

28 Hermans MP, Elisaf M, Michel G, et al. Benchmarking is associated with improved quality of care in type 2 diabetes: the OPTIMISE randomized, controlled trial. Diabetes Care 2013;36:3388-95.

29 Leite R, Leite RGOF, Mendes AL, et al. Effectiveness of strategies for nutritional therapy for patients with type 2 diabetes and/or hypertension in primary care: protocol of a systematic review of randomised controlled trials. BMJ Open 2019;9:e030450.

30 Ouzzani M, Hammady H, Fedorowicz Z, et al. Rayyan - a web and mobile app for systematic reviews. Syst Rev 2016;5:210.

31 Higgins JPT SJ, Savović J, Page MJ. A revised tool for assessing risk of bias in randomized trials. In: Chandler J, McKenzie J, Boutron I, eds. Cochrane methods. Cochrane database of systematic reviews, 2016.

32 Collaboration TC. Cochrane Handbook for systematic reviews of interventions version 5.1.0, 2011

33 Furukawa TA, Barbui C, Cipriani A, et al. Imputing missing standard deviations in meta-analyses can provide accurate results. J Clin Epidemiol 2006:59:7-10.

34 Salanti G, Marinho V, Higgins JPT. A case study of multipletreatments meta-analysis demonstrates that covariates should be considered. J Clin Epidemiol 2009;62:857-64.

35 Rücker G, Schwarzer G. Reduce dimension or reduce weights? Comparing two approaches to multi-arm studies in network metaanalysis. Stat Med 2014:33:4353-69.

36 Salanti G, Ades AE, loannidis JPA. Graphical methods and numerica summaries for presenting results from multiple-treatment metaanalysis: an overview and tutorial. J Clin Epidemiol 2011;64:163-71.

37 Veroniki AA, Straus SE, Fyraridis A, et al. The rank-heat plot is a novel way to present the results from a network meta-analysis including multiple outcomes. J Clin Epidemiol 2016;76:193-9.

38 Turner RM, Davey J, Clarke MJ, et al. Predicting the extent of heterogeneity in meta-analysis, using empirical data from the Cochrane database of systematic reviews. Int J Epidemiol 2012:41:818-27.

39 Rhodes KM, Turner RM, Higgins JPT. Predictive distributions were developed for the extent of heterogeneity in meta-analyses of continuous outcome data. J Clin Epidemiol 2015;68:52-60.

40 Higgins JPT, Thompson SG. Quantifying heterogeneity in a metaanalysis. Stat Med 2002;21:1539-58.

41 Higgins JPTet al. Measuring inconsistency in meta-analyses. BMJ 2003;327:557-60.

42 Lambert PC, Sutton AJ, Burton PR, et al. How vague is vague? A simulation study of the impact of the use of vague prior distributions in MCMC using WinBUGS. Stat Med 2005;24:2401-28.

43 White IR, Barrett JK, Jackson D, et al. Consistency and inconsistency in network meta-analysis: model estimation using multivariate metaregression. Res Synth Methods 2012;3:111-25.

44 Higgins JP, Jackson D, Barrett JK, et al. Consistency and inconsistency in network meta-analysis: concepts and models for multi-arm studies. Res Synth Methods 2012;3:98-110.

45 Veroniki AA, Mavridis D, Higgins JPT, et al. Characteristics of a loop of evidence that affect detection and estimation of inconsistency: a simulation study. BMC Med Res Methodol 2014;14:106.

46 Veroniki AA, Vasiliadis HS, Higgins JP, et al. Evaluation of inconsistency in networks of interventions. Int J Epidemiol 2013;42:332-45.

47 Begg CB, Mazumdar M. Operating characteristics of a RANK correlation test for publication bias. Biometrics 1994;50:1088-101. 
48 Egger M, Smith GD, Schneider M, et al. Bias in meta-analysis detected by a simple, graphical test. BMJ 1997;315:629-34.

49 Salanti G, Del Giovane C, Chaimani A, et al. Evaluating the quality of evidence from a network meta-analysis. PLoS One 2014;9:e99682.
50 Loveman E, Frampton G, Clegg A. The clinical effectiveness of diabetes education models for type 2 diabetes: a systematic review. Health Technol Assess 2008;12:1-116. 